Artigo Original

Original Article

Fernanda Dreux Miranda Fernandes ${ }^{1}$ Cibelle Albuquerque de la Higuera Amato ${ }^{\top}$

Descritores

Transtorno autista

Fonoaudiologia

Terapia comportamental

Terapia de linguagem

Keywords

Autistic disorder

Speech language and hearing sciences

Behavior therapy

Language therapy

Endereço para correspondência: Fernanda Dreux Miranda Fernandes R. do Mangericão, 301, Granja Vianna, Cotia (SP), Brasil, CEP: 06706-240.

E-mail: fernandadreux@usp.br

\section{Análise de Comportamento Aplicada e Distúrbios do Espectro do Autismo: revisão de literatura}

\section{Applied Behavior Analysis and Autism Spectrum Disorders: literature review}

\section{RESUMO}

Objetivo: Realizar uma revisão sistemática da literatura envolvendo as propostas de terapia baseada na análise de comportamento aplicada (ABA) dirigida a pessoas portadoras de distúrbios do espectro do autismo (DEA), contribuindo, dessa forma, para uma prática efetivamente baseada em evidências. Estratégia de pesquisa: As bases de dados Web of Science, Medline, SciELO e Lilacs foram consultadas para o levantamento das referências bibliográficas publicadas nos últimos cinco anos. Critérios de seleção: Foram selecionados os artigos publicados em periódicos com revisão por pares. Foram utilizados como critérios de exclusão o idioma, o tipo de artigo, o tema e os artigos repetidos. Essa seleção resultou em 52 artigos, que foram analisados na íntegra. Análise dos dados: Foram consideradas as informações referentes a autor, periódico e data; título; tema e abordagem; casuística; critérios de inclusão e exclusão e conclusões. Resultados: Os artigos abordam processos de intervenção, revisões de literatura, formação profissional e a contribuição dos pais no processo de intervenção. Apenas quatro artigos relatam a contribuição dos pais na aplicação dos princípios da ABA no ambiente doméstico. Os estudos sobre formação profissional enfatizam a valorização da formação especializada. A maioria das revisões de literatura conclui que os processos de intervenção são controversos, caros e dependentes de fatores externos. Embora artigos que relatam processos de intervenção envolvam 663 participantes, não é possível a realização de meta-análise devido à ausência de critérios de inclusão e caracterização comparáveis. Conclusão: Não há evidência suficiente para corroborar a preponderância da ABA sobre outras alternativas.

\begin{abstract}
Purpose: Systematic literature review about Applied Behavior Analysis (ABA) proposals directed towards persons with Autistic Spectrum Disorders aiming to contribute to a truly evidence-based practice. Research strategy: References from the last five years were obtained from the Web of Science, Medline, SciELO and Lilacs databases. Selection criteria: Papers published in peer-reviewed journals were selected. Exclusion criteria were language, type of paper, theme and repeated papers. This selection resulted in 52 articles that were completely analyzed. Data analysis: Information regarding author, journal and date; title; theme and approach; casuistic; inclusion and exclusion criteria and conclusion was considered. Results: The papers refer to intervention processes, literature reviews, professional education, and parents' contributions to the intervention programs. Only four papers report the parents' role in the use of ABA principles at home. Studies about Professional education emphasize the specialized education. Most of the literature review papers conclude that the intervention programs are controversial, expensive and dependent of external variables. Although the articles describing intervention processes include 663 participants, a meta-analysis is not possible due to the lack of comparable inclusion and characterization criteria. Conclusion: There is not enough evidence of ABA's preponderance over other alternatives.
\end{abstract}

Recebido em: 07/06/2013

Trabalho realizado no Laboratório de Investigação Fonoaudiológica nos Distúrbios do Espectro do Autismo, Curso de Fonoaudiologia, Faculdade de Medicina, Universidade de São Paulo - USP - São Paulo (SP), Brasil. (1) Laboratório de Investigação Fonoaudiológica nos Distúrbios do Espectro do Autismo, Curso de Fonoaudiologia, Faculdade de Medicina, Universidade de São Paulo - USP - São Paulo (SP), Brasil.

Conflito de interesse: nada a declarar.

Aceito em: 12/06/2013 


\section{INTRODUÇÃO}

As abordagens terapêuticas e educacionais dirigidas a pessoas com distúrbios incluídos no espectro do autismo (DEA) têm sido objeto de debates frequentemente contaminados por ideologias, modismos e política, muitas vezes desconsiderando ou desvalorizando as evidências científicas a respeito de sua eficiência e validade social ${ }^{(1,2)}$. Propostas de intervenção baseadas no modelo da análise de comportamento aplicada (Applied Behavior Analysis - ABA) têm sido frequentemente mencionadas como o único modelo com resultados cientificamente comprovados ${ }^{(2-4)}$.

Programas baseados na ABA exigem a verificação detalhada dos fatores ambientais e de sua interferência nos comportamentos da criança com DEA, buscando a identificação dos determinantes do comportamento e dos fatores que provavelmente resultarão na sua repetição. Essas informações são essenciais para o delineamento e acompanhamento dos processos de intervenção ${ }^{(2,3)}$. Os programas frequentemente incluem as habilidades verbais e de comunicação em níveis de intensidade da intervenção semelhantes aos destinados às habilidades cognitivas e acadêmicas e às dificuldades de comportamento ${ }^{(2,3)}$. Além disso, a utilização estrita dos princípios da ABA e a formação específica e consistente dos terapeutas também são consideradas elementos essenciais para o sucesso da proposta ${ }^{(4,5)}$. A participação dos pais, proporcionando uma estimulação mais intensiva no ambiente doméstico, frequentemente é mencionada como um dos pontos a favor da utilização das abordagens de $\mathrm{ABA}^{(6,7)}$.

Recentemente alguns estudos admitem a necessidade de mais pesquisas a respeito dos resultados de processos de intervenção direcionados a "comportamentos de níveis superiores" (como a fala e a insistência na mesmice) ${ }^{(8,9)}$. Nesse sentido, a terapia "cognitivo-comportamental" tem sido proposta como uma alternativa para a continuidade do processo de intervenção comportamental estrita ${ }^{(10)}$.

A inclusão dos "comportamentos comunicativos", como parte dos objetivos das intervenções baseadas na ABA para crianças com $\mathrm{DEA}^{(11)}$, tem levado profissionais de outras áreas a abordarem a fala, de forma frequentemente antagônica ou conflitante com a terapia de linguagem ${ }^{(12)}$. Por outro lado, alguns fonoaudiólogos têm utilizado fundamentos da ABA na terapia fonoaudiológica dirigida a crianças com DEA. Dessa forma, é relevante que a literatura que relata os resultados desse tipo de intervenção seja revisada criticamente.

\section{OBJETIVOS}

Realizar uma revisão sistemática da literatura envolvendo as propostas de terapia baseada na ABA dirigida a pessoas portadoras de DEA, contribuindo dessa forma para uma pratica efetivamente baseada em evidências.

\section{ESTRATÉGIA DE PESQUISA}

As bases de dados Web of Science, Medline, SciELO e Lilacs foram consultadas, utilizando os termos "autism,
ABA"; "autism, applied behavior analysis"; "autismo, ABA" e "autismo, análise de comportamento aplicada", para o levantamento das referências bibliográficas publicadas nos últimos cinco anos.

\section{CRITÉRIOS DE SELEÇÃO}

O primeiro levantamento de dados identificou 672 artigos; dentre esses, foram selecionados mecanicamente apenas aqueles publicados em periódicos com revisão por pares, resultando em 427 artigos. A partir daí, a revisão manual, pela leitura do título e do resumo de cada artigo, utilizou como critérios de exclusão o idioma (incluindo apenas as publicações em inglês, português e espanhol), o tipo de artigo (excluindo resenhas, resumos, editoriais, cartas ao editor e tutoriais) e o tema (excluindo artigos direcionados a financiamento do tratamento, recursos dos sistemas de saúde, acesso ao atendimento, oferta de atendimento a familiares de militares, questões legais, modelos animais e outros temas em saúde mental). Nesse processo foram também excluídos os artigos repetidos. Essa seleção resultou em 52 artigos, que foram analisados na íntegra.

\section{ANÁLISE DOS DADOS}

Os artigos selecionados para esta revisão foram sintetizados em uma planilha, para o registro de informações referentes a autor, periódico e data; título; tema e abordagem; casuística; critérios de inclusão e exclusão quando apropriado; e conclusões do estudo.

\section{RESULTADOS}

Os 52 artigos analisados foram publicados em 25 diferentes periódicos. A distribuição dessa publicação está apresentada no Quadro 1 e revela uma abrangência expressiva, que inclui periódicos especializados em autismo, pediatria, estudos comportamentais, distúrbios de comunicação, psicologia e até odontologia. A análise do fator de impacto revela que 64\% dos periódicos têm esse índice calculado e que há grande variação nos indicadores. Enquanto o maior fator de impacto identificado é superior a 9 , o menor deles está abaixo de 0,5. Há 20 artigos publicados nos seis periódicos com fator de impacto superior a 2, quatro artigos em periódicos com fator de impacto acima de 1 e 13 artigos publicados em periódicos com índice de impacto inferior a 1. Esses resultados revelam, mais uma vez, a abrangência e o interesse despertados por essa área de estudo.

No Quadro 2, pode-se observar que $43 \%$ dos artigos abordam a análise de processos de intervenção, $32 \%$ são revisões de literatura, $19 \%$ referem-se à análise da formação e atuação de profissionais e $6 \%$ estão focalizados na atuação e na contribuição dos pais para o processo de intervenção.

Pode-se observar também que não existe uma repetição significativa nos nomes de autores ou de grupos de pesquisadores. O nome de apenas um autor aparece em quatro artigos, outro pesquisador é autor de três artigos e outros quatro estão entre os autores de dois artigos cada um. Isso parece indicar, 
Quadro 1. Índice de impacto e número de artigos por periódico

\begin{tabular}{|c|c|c|}
\hline Título do periódico & № de artigos & FI-JCR \\
\hline $\begin{array}{l}\text { Journal of Autism and Developmental } \\
\text { Disorders }\end{array}$ & 14 & 3,34 \\
\hline Focus on Autism and Other & 1 & 1,048 \\
\hline Developmental Disabilities & 1 & 9.111 \\
\hline Autism & 2 & 2,265 \\
\hline Research on Developmental Disabilities & 4 & 0,88 \\
\hline Journal of Intellectual Disability Research & 1 & 1,877 \\
\hline Behavior Modification & 2 & - \\
\hline Education and Treatment of Children & 1 & 0,47 \\
\hline $\begin{array}{l}\text { The American Journal of Occupational } \\
\text { Therapy }\end{array}$ & 1 & 1,7 \\
\hline Journal of Behavioral Education & 2 & 0,51 \\
\hline Journal of Pediatrics & 1 & 4,115 \\
\hline $\begin{array}{l}\text { Journal of Speech Language Pathology } \\
\text { and Applied Behavior Analysis }\end{array}$ & 3 & 1,19 \\
\hline Teaching Exceptional Children & 2 & - \\
\hline $\begin{array}{l}\text { Journal of the American Dental } \\
\text { Association }\end{array}$ & 1 & 2,195 \\
\hline British Journal of Special Education & 1 & - \\
\hline The Psychological Record & 1 & - \\
\hline Infants \& Young Children & 1 & 0,91 \\
\hline The Behavior Bulletin & 1 & - \\
\hline $\begin{array}{l}\text { International Journal of Behavioral } \\
\text { Consultation and Therapy }\end{array}$ & 2 & - \\
\hline The Behavior Analyst Today & 3 & 0,491 \\
\hline $\begin{array}{l}\text { The Journal of Early and Intensive } \\
\text { Behavioral Intervention }\end{array}$ & 2 & - \\
\hline Clinical Psychology Review & 1 & 8,392 \\
\hline Behavioral Interventions & 2 & 0,8 \\
\hline $\begin{array}{l}\text { Revista da Facultad de Medicina de } \\
\text { a.Universidad Nacional de Colombia }\end{array}$ & 1 & - \\
\hline Current Topics in Review & 1 & - \\
\hline
\end{tabular}

Legenda: FI-JCR: fator de impacto no Journal Citation Report

mais uma vez, a abrangência do tema e dos grupos de pesquisa envolvidos com essa proposta.

Embora diversos estudos apontem para a importância da contribuição dos pais na aplicação dos princípios da ABA no ambiente doméstico ${ }^{(2,4,5,8,13)}$, esta revisão da literatura encontrou poucos resultados científicos dessa proposta. Uma pesquisa ${ }^{(9)}$ com 53 pais de crianças com DEA relatou que todos foram positivos quanto aos resultados de uma proposta de dois anos de intervenção intensiva. Um trabalho recente ${ }^{(14)}$ investigou a opinião de pais e irmãos de crianças com DEA a respeito dos resultados de um programa de intervenção no lar. A autora concluiu que os resultados foram predominantemente neutros, sendo que os pais se mostraram mais otimistas do que os irmãos em relação aos resultados da proposta, mas mesmo eles não verificaram benefícios para os irmãos que pudessem ser associados à intervenção comportamental junto às crianças com DEA. O outro artigo revisado ${ }^{(7)}$ relata a experiência de seis mães que optaram por estudar profundamente a metodologia $\mathrm{ABA}$, tornando-se terapeutas credenciadas para essa atividade; mas não é possível generalizar o significado dessa experiência para todas elas, nem antecipar esse significado para outros pais que optem por esse caminho.

Os artigos referentes à educação profissional abordam reflexões a respeito de educação à distância, adaptação de programas a contextos linguísticos diferentes, uso de vídeos para modelagem de comportamentos e supervisão. O número de participantes em todos esses estudos chega a 253 profissionais (terapeutas e educadores), mas a única posição que pode ser generalizada envolve a valorização da formação especializada ${ }^{(3,7)}$. A forma de coleta de dados inclui a aplicação de questionários e entrevistas, mas em geral há pouca informação a respeito da formação do grupo de profissionais pesquisados.

$\mathrm{O}$ segundo maior grupo de artigos envolve os que realizaram uma revisão da literatura. Destes, dois apresentam meta-análises que somam 200 participantes e ambos concluem que não há evidencia nítida de que a $\mathrm{ABA}$ apresente uma vantagem relevante em relação a outras propostas de intervenção ${ }^{(1)}$. Um terceiro estudo ${ }^{(15)}$ revisou cinco estudos de meta-análise a respeito de intervenção comportamental intensiva e identificou limitação nesses estudos devido às descrições inconsistentes dos participantes, métodos, critérios de inclusão e processos de intervenção. As posições resultantes das revisões de literatura analisadas também não são consistentes. Os resultados dos processos de intervenção são apontados como controversos, caros e dependentes de fatores externos (como o ambiente, a quantidade de treinamento oferecido e a interação com medicamentos) por sete artigos, que também chamam a atenção para a necessidade de estudos mais controlados a respeito dos resultados desses processos de intervenção $0^{(2,13)}$. Outras três revisões da literatura ${ }^{(10)}$ apresentam posições nitidamente contrárias à utilização da $\mathrm{ABA}$ como o principal programa de intervenção direcionado a indivíduos com DEA, considerando essa abordagem ineficiente e sem sustentação científica.

Os artigos que relatam processos de intervenção utilizando os princípios da ABA representam $44 \%$ do conjunto de artigos revisados. O primeiro aspecto considerado foi o número de sujeitos de cada estudo, que varia de estudos com apenas um participante com $\operatorname{DEA}^{(16,17)}$ a outro artigo que relata os resultados de intervenções realizadas com 208 participantes $^{(18)}$. Embora esses artigos envolvam um total de 663 participantes, não é possível a realização de meta-análise devido à ausência de critérios de inclusão comparáveis ou mesmo de uma descrição mais completa a respeito das características de idade, quadro clínico e nível de desenvolvimento dos participantes dos processos relatados. Em relação à idade dos participantes, ocorre grande variação na faixa etária abrangida, que inclui participantes de menos de 36 meses e adolescentes. Além disso, essa característica também é descrita com diferentes graus de especificidade, com alguns artigos descrevendo faixas etárias como "entre 2 anos e 4 meses e 6 anos e 2 meses" ${ }^{(11)}$ e outros que se referem apenas a "crianças" (19) ou "adolescentes"(20).

Em algumas pesquisas, os critérios de inclusão são específicos e detalhados, com a utilização de escalas de desenvolvimento, instrumentos de diagnóstico e caracterização sociodemográfica ${ }^{(5,11)}$. Em outros estudos, entretanto, a inclusão dos participantes nos processos de intervenção envolveu critérios de conveniência, como frequentar uma determinada escola ou estar 
Quadro 2. Temática e casuística dos artigos revisados

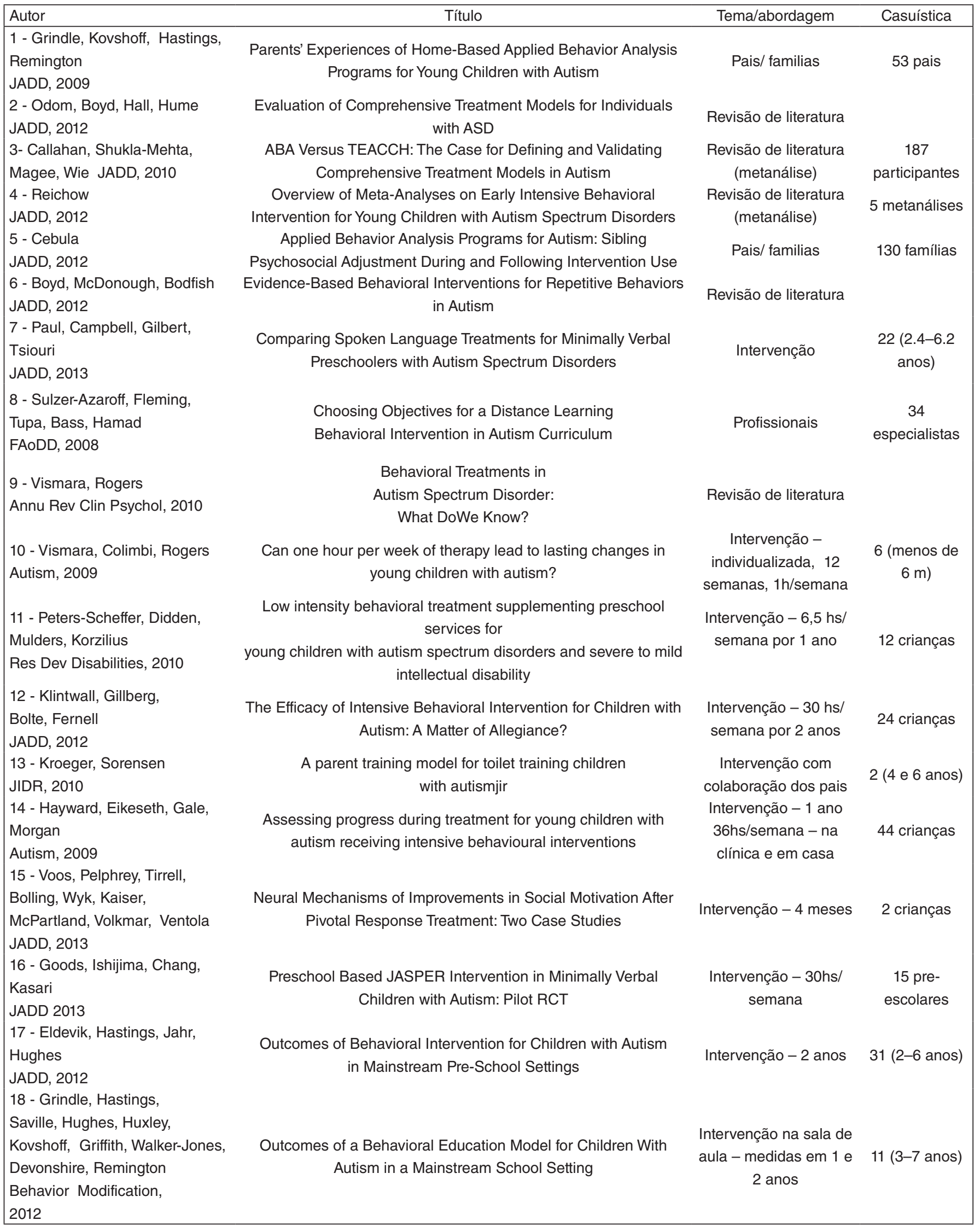

Continua... 
Quadro 2. Continuação

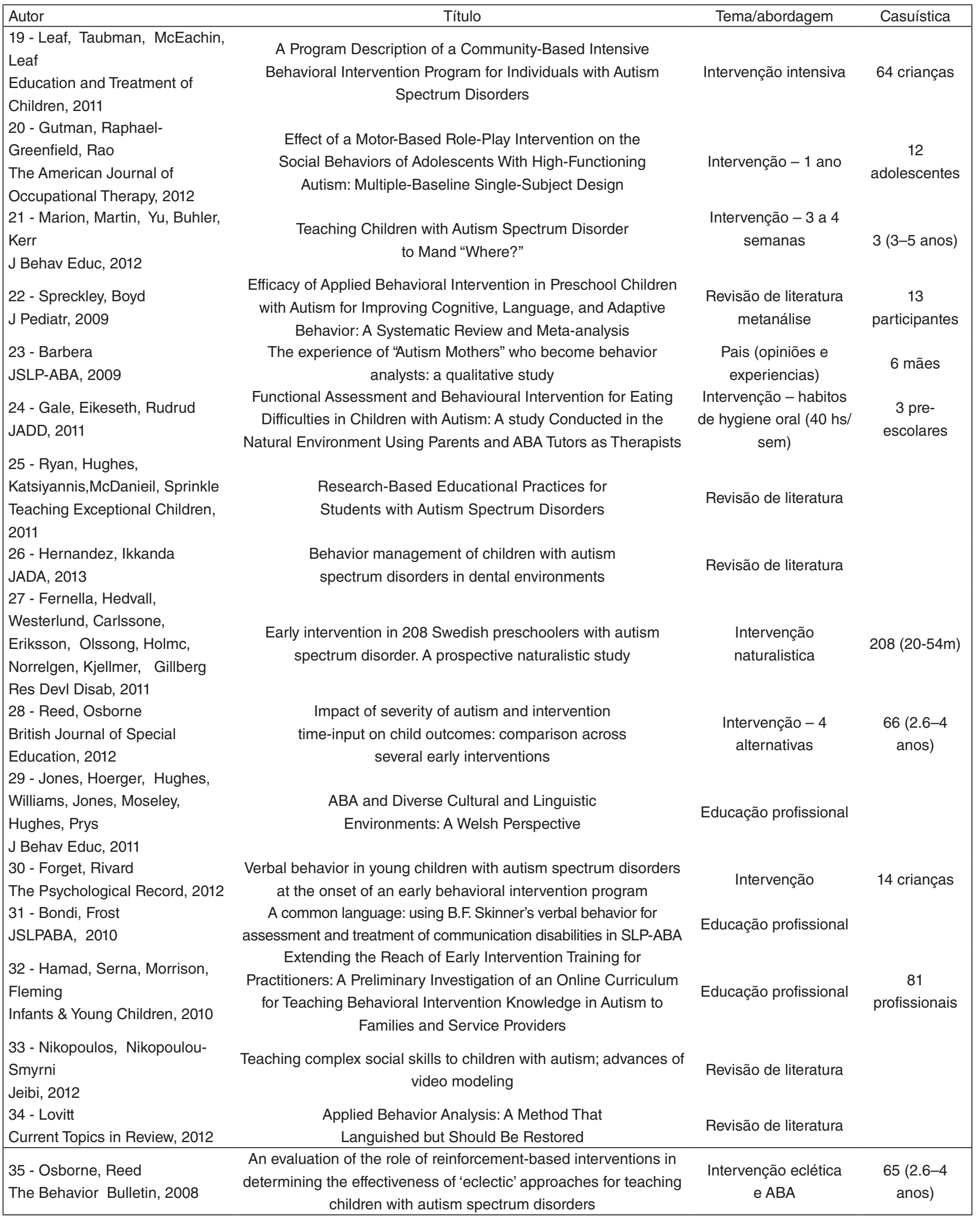

Continua... 
Quadro 2. Continuação

\begin{tabular}{|c|c|c|c|}
\hline Autor & Título & Tema/abordagem & Casuística \\
\hline $\begin{array}{l}36 \text { - Vismara, Young, Stahmer, } \\
\text { Griffith, Rogers } \\
\text { JADD, } 2009\end{array}$ & $\begin{array}{c}\text { Dissemination of Evidence-Based Practice: Can We Train } \\
\text { Therapists from a Distance? }\end{array}$ & Educação profissional & $\begin{array}{l}10 \\
\text { profissionais }\end{array}$ \\
\hline $\begin{array}{l}37 \text { - Wolfe, Condo, Hardaway } \\
\text { Teaching Exceptional Children, } \\
2009\end{array}$ & $\begin{array}{l}\text { Sociosexuality Education for Persons With Autism Spectrum } \\
\text { Disorders Using Principles of Applied Behavior Analysis }\end{array}$ & Educação profissional & \\
\hline $\begin{array}{l}38 \text { - Dyer } \\
\text { JSLPABA, } 2009\end{array}$ & $\begin{array}{c}\text { Clinical application of speech intelligibility research: the River } \\
\text { Street Autism Program at Coltsville }\end{array}$ & Revisão de literatura & \\
\hline $\begin{array}{l}39 \text { - Healy, Keohane, Leader, } \\
\text { Lydon, } \\
\text { JEIBI, } 2010\end{array}$ & $\begin{array}{l}\text { The effects of intensive tact instruction on three verbal operants in } \\
\text { non-instructional settings for two children with autism }\end{array}$ & $\begin{array}{l}\text { Intervenção (15 } \\
\text { minutos) }\end{array}$ & 2 crianças \\
\hline $\begin{array}{l}40 \text { - Luiselli, MaGee, Sperry, } \\
\text { St. Amand. } \\
\text { IJBCT, } 2008\end{array}$ & $\begin{array}{c}\text { Group training of applied behavior analysis (ABA) knowledge } \\
\text { competencies to community-based service providers for adults } \\
\text { with developmental disabilities }\end{array}$ & Educação profissional & $\begin{array}{l}47 \\
\text { profissionais }\end{array}$ \\
\hline $\begin{array}{l}41 \text { - Gibson, Grey, Hastings. } \\
\text { JADD, } 2009\end{array}$ & $\begin{array}{c}\text { Supervisor Support as a Predictor of Burnout and Therapeutic } \\
\text { Self-Efficacy in Therapists Working in ABA Schools }\end{array}$ & Educação profissional & $\begin{array}{l}81 \\
\text { profissionais }\end{array}$ \\
\hline $\begin{array}{l}42 \text { - DelPizzo-Cheng, LaRue, } \\
\text { Sloman, Weiss } \\
\text { The behavior analyst today, } 2010\end{array}$ & $\begin{array}{c}\text { ABA and PBS: the dangers in creating artificial dichotomies in } \\
\text { behavioral intervention }\end{array}$ & Educação profissional & \\
\hline $\begin{array}{l}43 \text { - Schreck, Mazur } \\
\text { Behav Interventions, } 2008\end{array}$ & $\begin{array}{l}\text { Behavior analyst use of and beliefs in } \\
\text { Treatments for people with autism }\end{array}$ & Educação profissional & \\
\hline $\begin{array}{l}44 \text { - Brock, Bruneau, Davis, } \\
\text { McNulty, Rosswurm, Zane } \\
\text { The behavior analyst today, } 2010\end{array}$ & $\begin{array}{l}\text { Efficiency of forced choice preference assessment: comparing } \\
\text { multiple presentation techniques }\end{array}$ & Intervenção & 6 (8-12 anos) \\
\hline $\begin{array}{l}45 \text { - Eldevik, Jahr, Eikeseth, } \\
\text { Hastings, Hughes } \\
\text { Behavior Modification, } 2010\end{array}$ & $\begin{array}{l}\text { Cognitive and Adaptive Behavior Outcomes of Behavioral } \\
\text { Intervention for Young Children With Intellectual Disability }\end{array}$ & $\begin{array}{c}\text { Intervenção } \\
\text { (comparando modelos) }\end{array}$ & 25 crianças \\
\hline $\begin{array}{l}46 \text { - Healy, Kenny, Leader, } \\
\text { O'Connor } \\
\text { The J. of Early and Intensive } \\
\text { Behavioral Intervention, } 2008\end{array}$ & Three years of intensive applied behavior analysis: a case study & $\begin{array}{l}\text { Intervenção (na } \\
\text { escola) }\end{array}$ & $1(2 \mathrm{a}, 10 \mathrm{~m})$ \\
\hline 47 - Virués-Ortega & Applied behavior analytic intervention for autism in early & & \\
\hline $\begin{array}{l}\text { Clinical Psychology Review, } \\
2010\end{array}$ & $\begin{array}{c}\text { childhood: Meta-analysis, meta-regression and dose-response } \\
\text { meta-analysis of multiple outcomes }\end{array}$ & Revisão de literatura & \\
\hline $\begin{array}{l}48 \text { - DeRosa, Gadaire, Kelley. } \\
\text { The behavior analyst today, } 2011\end{array}$ & $\begin{array}{l}\text { Research needed for focusing on additional generality of applied } \\
\text { behavior analysis }\end{array}$ & Revisão de literatura & \\
\hline $\begin{array}{l}49 \text {-Schreck, Miller. Behavioral } \\
\text { Interventions, } 2010\end{array}$ & How to Behave Ethically in a World of Fads & Revisão de literatura & \\
\hline $\begin{array}{l}50 \text { - Axelrod, Bloh. } \\
\text { The Journal of Early } \\
\text { and Intensive Behavioral } \\
\text { Intervention, } 2008\end{array}$ & $\begin{array}{l}\text { IDEIA and the means to change behavior should be enough: } \\
\text { growing support for using applied behavior analysis in the } \\
\text { classroom }\end{array}$ & Revisão de literatura & \\
\hline $\begin{array}{l}51 \text { - Barnes-Holme, Murphy } \\
\text { The Psychological Record, } 2009\end{array}$ & $\begin{array}{l}\text { Establishing derived manding for specific amounts with three } \\
\text { children: an attempt at Synthesizing Skinner's Verbal Behavior } \\
\text { with relational frame theory }\end{array}$ & $\begin{array}{l}\text { Intervenção (2 normais } \\
\text { com } 9 \text { e } 10 \text { anos e I } \\
\text { DEA com } 4 \text { anos }\end{array}$ & 3 crianças \\
\hline $\begin{array}{l}52 \text { - Piñeros-Ortiz, Toro-Herrera } \\
\text { Rev fac med u nal Colombia. } \\
2012\end{array}$ & $\begin{array}{l}\text { General concepts concerning applied behavior analysis (ABA) in } \\
\text { children suffering autistic spectrum disorders (ASD) }\end{array}$ & Revisão de literatura & \\
\hline
\end{tabular}


incluído em um programa de intervenção destinado às crianças de uma determinada cidade ou comunidade ${ }^{(21)}$. Seguramente, determinar critérios de verificação dos resultados de intervenção que possam ser eficientes para avaliar processos tão diferentes é um desafio que ainda não foi solucionado.

Essa mesma diversidade pode ser observada no que diz respeito ao tempo envolvido no programa de intervenção. No Quadro 2, é possível observar que os processos descritos duraram entre três e quatro semanas, 12 semanas, oito meses, um ano ou dois anos, em programas de uma hora por semana e até 30 ou 36 horas por semana. Os processos de treinamento ocorreram principalmente na escola ${ }^{(21)}$ (na própria sala de aula ou em alguma sala silenciosa), em clínicas ${ }^{(8)}$ ou na casa dos participantes.

Os objetivos dos programas de intervenção variam entre imitação motora ${ }^{(11)}$, habilidades adaptativas ${ }^{(19)}$, treino de toalete, habilidades motoras, sociais e de linguagem ${ }^{(5,9,20,21)}$. Alguns $\operatorname{artigos}^{(5,9,16)}$ relatam resultados de sucesso em abordagens de ABA, com progressos relevantes para todos os participantes em diferentes áreas, especialmente em programas de intervenção precoce intensiva. Esses estudos, entretanto, em geral não incluem grupos controle. Estudos que compararam programas com abordagem ABA a modelos "naturalísticos" ou abrangentes ${ }^{(8,11,18,19,21)}$ concluíram que não há diferença relevante nos resultados de ambas as abordagens, especialmente no que se refere à severidade dos sintomas de autismo.

A relação direta entre o número de horas de treinamento e os resultados do processo, relatado como bem sucedido em todos os casos, é mencionada por 12 artigos, representando um total de 182 participantes. Apenas um estudo ${ }^{(22)}$, com seis participantes, concluiu que um programa com uma hora semanal de intervenção proporciona resultados satisfatórios. Os outros dez artigos, que compararam os resultados de programas de intervenção com abordagem de ABA a diferentes propostas terapêuticas, sem verificar diferenças significativas entre os resultados, somam um total de 453 participantes.

\section{CONCLUSÃO}

Esta revisão compilou um número relevante de artigos que descrevem estudos e outras revisões de literatura a respeito de uma proposta de intervenção que é frequentemente mencionada como a única abordagem terapêutica que apresenta resultados cientificamente comprovados para indivíduos com DEA. O número de periódicos revisados e sua abrangência temática indicam a imparcialidade do levantamento. Os estudos que compararam a abordagem de ABA a outras propostas levam às mesmas conclusões de outras revisões de literatura, ou seja, não há evidencia suficiente para corroborar a preponderância da ABA sobre outras alternativas.

A busca por procedimentos de intervenção que sejam eficazes, socialmente relevantes e economicamente viáveis é fundamental para o aperfeiçoamento do atendimento aos indivíduos com DEA. Entretanto, a análise do material indica que há necessidade de estudos controlados, com casuística relevante e critérios claros de inclusão e de avaliação dos resultados, para que qualquer proposta de intervenção possa ser considerada mais eficiente ou produtiva do que outras.
A opção por um método ou procedimento terapêutico deve ser fundamentada em informações claras a respeito de seus princípios, técnicas e expectativas de resultados e também das alternativas disponíveis. Espera-se que esta revisão contribua para que o fonoaudiólogo possa realizar escolhas que sejam cada vez mais baseadas em evidências científicas, mesmo que isso signifique a admissão de que não existem respostas únicas que se apliquem a todos os indivíduos com DEA. Esse processo deve incluir orientações e informações às famílias quanto às alternativas disponíveis, suas vantagens e limitações.

*FDMF foi responsável pela coleta do material e redação do texto; CAHA colaborou com a coleta do material, revisão e formatação do texto.

\section{REFERÊNCIAS}

1. Callahan K, Shukla-Mehta S, Magee S, Wie M. ABA versus TEACCH: the case for defining and validating comprehensive treatment models in autism. J Autism Develop Disord. 2010;40:74-8.

2. Vismara LA, Rogers S. Behavioral treatments in Autism Spectrum Disorder: what do we know? Annu Rev Clin Psychol. 2010;6:447-68.

3. Sulzer-Azaroff B, Fleming R, Tupa M, Bass R, Hamad C. Choosing objectives for a distance learning behavioral intervention in autism curriculum. Focus Autism Other Dev Disabl. 2008;23:29-36.

4. Klintwall L, Gillberg C, Bölte S, Fernell E. The efficacy of intensive behavioral intervention for children with autism: a matter of allegiance? J Autism Dev Disord. 2012;42(2):139-40.

5. Eldevik S, Hastings RP, Jahr E, Hughes JC. Outcomes of behavioral intervention for children with autism in mainstream pre-school settings. J Autism Dev Disord. 2012;42(2):210-20.

6. Kroeger K, Sorensen R. A parent training model for toilet training children with autism. J Intellect Disabil Res. 2010;54(6):556-67.

7. Barbera ML. The experiences of "autism mothers" who become behavior analysts: a qualitative study. J Spe Lang Path Apl Behav Anal. 2009;4(1):56-73.

8. Hayward D, Eikeseth S, Gale C, Morgan S. Assessing progress during treatment for young children with autism receiving intensive behavioral interventions. Autism. 2009;13(6):613-33.

9. Grindle CF, Hastings RP, Saville M, Hughes JC, Huxley K, Kovshoff H, et al. Outcomes of a behavioral education model for children with autism in a mainstream school setting. Behav Modif. 2012;36(3):298-319.

10. Boyd BA, McDonough SG, Bodfish JW. Evidence-based behavioral interventions for repetitive behaviors in autism. J Autism Dev Disord. 2012;42(6):1236-48.

11. Paul R, Campbell D, Gilbert K, Tsiouri I. Comparing spoken language treatments for minimally verbal preschoolers with autism spectrum disorders. J Autism Dev Disord. 2013;43(2):418-31.

12. Kasari C, Paparella T, Freeman S, Jahromi L. Language outcome in autism: randomized comparison of joint attention and play interventions. J Consult Clin Psychology. 2008;76(1):125-37.

13. Odom SL, Boyd BA, Hall LJ, Hume K. Evaluation of comprehensive treatment models for individuals with autism spectrum disorders. J Autism Dev Disord. 2010;42(2):425-36.

14. Cebula KR. Applied behavior analysis programs for autism: sibling psychosocial adjustment during and following intervention use. J Autism Dev Disord. 2012;42(4):847-62.

15. Reichow B. Overview of meta-analyses on early intensive behavioral intervention for young children with autism spectrum disorders. J Autism Dev Disord. 2012;42(3):512-20.

16. Haely C, Kenny M, Leader K, O’Connor J. Three years of intensive applied behavior analysis: a case study. J Early Intens Behav Interv. 2008;5(1):4-23. 
17. Barnes-Holme D, Murphy C. Establishing derived manding for specific amounts with three children: an attempt at Synthesizing Skinner's Verbal Behavior with relational frame theory. Psychol Rec. 2009;59(1):75-9.

18. Fernell E, Hedvall Å, Westerlund J, Carlsson LH, Eriksson M, Olsson MB, et al. Early intervention in 208 Swedish preschoolers with autism spectrum disorder. A prospective naturalistic study. Res Dev Disabil. 2010;31(3):790-99.

19. Peters-Scheffer H, Didden P, Mulders C, Korzilius H. Low intensity behavioral treatment supplementing preschool services for young children with autism spectrum disorders and severe to mild intellectual disability. Res Dev Disabil. 2010;31(6):1678-84.
20. Gutman G, Greenfield R, Rao P. Effect of a motor-based role-play intervention on the social behaviors of adolescents with high-functioning autism: multiple-baseline single-subject design. Am J Occup Therapy. 2012;66(5):529-38.

21. Reed C, Osborne M. Impact of severity of autism and intervention timeinput on child outcomes: comparison across several early interventions. Brit J Spec Educ. 2012;39(3):130-6.

22. Vismara LA, Colombi C, Rogers S. Can one hour per week of therapy lead to lasting changes in Young children with autism? Autism. 2009;13(1):93-115. 